\title{
ICTIOFAUNA DO PARQUE ESTADUAL MATA DO XÉM-XÉM, BACIA DO RIO PARÁBBA DO NORTE, PARÁ́BA, BRASIL
}

\author{
ICTIOFAUNA OF THE STATE PARK MATA DEL XEM-XÉM, \\ BASIN OF THE RIVER PARAÍBA OF THE NORTH, PARAÍBA, BRAZIL
}

\author{
Telton Pedro Anselmo Ramos ${ }^{1}$ \\ Silvia Yasmin Lustosa Costa ${ }^{2}$ \\ Leonardo Oliveira Silva ${ }^{3}$
}

\section{Resumo}

Neste trabalho são apresentados dados referentes a um inventário da fauna de peixes do Parque Estadual Mata do Xem-Xém, uma Unidade de Conservação localizada no trecho inferior da bacia hidrográfica do rio Paraíba do Norte, sob domínio de Mata Atlântica, Estado da Paraíba, Brasil. Foram amostrados sete pontos de coleta em diferentes ambientes aquáticos, incluindo rios, riachos, e um lago, situados tanto no interior quanto no entorno da unidade. Foram registradas 15 espécies de peixes, das quais 13 são nativas e duas introduzidas (Oreochromis niloticus e Poecilia reticulata), distribuídas em quatro ordens, seis famílias e 13 gêneros. Embora tenha sido registrada apenas no lago, recomenda-se o controle da população de Oreochromis niloticus desta Unidade de conservação, em função das ameaças que a espécie pode representar à ictiofauna nativa e aos demais ecossistemas aquáticos da região.

Palavras-chave: Espécies exóticas; inventário; peixes de água doce; Unidade de Conservação.

\begin{abstract}
Data referring to a survey of the fish fauna Parque Estadual Mata do Xem-Xém, a Conservation Unity located in the lower reaches of the Paraíba do Norte river basin under the Atlantic Forest, State of Paraíba are presented in this study. Seven sites were sampled in several aquatic environments, including rivers, streams, and a lake situated inside and in the nearby area of the unity. Fifteen species of fish were registered, 13 native and 2 introduced (Oreochromis niloticus and Poecilia reticulata), distributed in four Orders, six Families and 13 Genera. Although Oreochromis niloticus was recorded only on the lake, their eradication from the conservation unity is recommended, considering the threats that the species may represent for

1 Universidade Estadual da Paraíba, Departamento de Biologia/CCBS, Laboratório de Ecologia Aquática, Campus universitário, CEP 58109-753, Campina Grande, PB, Brasil.

2 Universidade Federal do Rio Grande do Norte, Centro de Biociências, Departamento de Botânica e Zoologia, Laboratório de Ictiologia Sistemática e Evolutiva, Campus Central, Lagoa Nova, CEP 590789oo, Natal, RN, Brasil.

3 Universidade Federal da Bahia, Instituto de Biologia, Rua Barão de Geremoabo, 147, Ondina, CEP 40170-290, Salvador, BA, Brasil.
\end{abstract}


the native ichthyofauna and for the other aquatic ecosystems of the region.

Key words: Exotic species; inventory; freshwater fish; Conservation Unit.

\section{INTRODUÇÃO}

A ação humana sobre os ecossistemas terrestres e aquáticos tem se intensificado dia após dia, sendo resultado de uma necessidade constante de suprir uma população que só cresce. Esse crescimento, tem promovido destruição e fragmentação de áreas de florestas (Odum 1988; Albagli 2001), com impactos que têm afetado e comprometido a integridade da Mata Atlântica, que é um dos 25 hotspots mundiais de biodiversidade (Tabarelli et al. 2005). Diante desse cenário, a criação e manutenção de Unidades de Conservação (UC) têm sido incentivadas. De maneira geral, estas unidades atuam na mitigação de impactos ambientais nos biomas a longo prazo, pelo menos para preservar parte da biodiversidade atual para gerações futuras (MMA 2019). Isso tornou-se possível, graças à criação do Sistema Nacional de Conservação da Natureza (SNUC), com a promulgação da Lei no 9.985, de 18 de julho de 2000.

Atualmente, em território nacional, existem 2.309 Unidades de Conservação, nas esferas Federais (1.004), Estaduais (964) e Municipais (341) (CNUC/MMA, 2019). Desse total, 914 UCs estão localizadas em áreas de Mata Atlântica (Pinto et al. 2018). Particularmente para o estado da Paraíba, os resquícios de Mata Atlântica estão protegidos por algumas Unidades de Conservação, como o Parque Estadual (PE) Mata do Xem-Xém, que protege um total de 182 hectares de mata (SEMARH 2016).

O PE Mata do Xem-Xém possui parte de seus limites em uma área de nascentes do rio do Meio, que é um afluente do rio Paraíba do Norte. A bacia do rio Paraíba do Norte possui toda sua drenagem dentro do Estado da Paraíba, sendo uma das principais bacias que compõem a ecorregião Nordeste/Caatinga (Abell et al. 2008). Atualmente, são listadas 47 espécies de peixes de água doce para a bacia do rio Paraíba do Norte. A fauna aquática desta bacia atualmente é uma preocupação por parte dos pesquisadores, devido a transposição do São Francisco, que pode impactar a ictiofauna residente da bacia (Ramos et al. 2018).

Conhecer a ictiofauna de uma determinada bacia hidrográfica ou sub-bacia pode servir de base para caracterização ambiental, como indicação de degradação do ambiente (Ramos 2012). Ademais, o conhecimento da ictiofauna de uma área como o PE Mata do Xem-Xém pode ajudar a entender a história dos peixes da Mata Atlântica, isto se mostra de extrema relevância tendo em vista a trágica perda de elementos da fauna e da flora do bioma (Menezes et al. 2007). Dessa maneira, objetivamos com este trabalho fornecer a lista de espécies de peixes de água doce ocorrentes nos rios que drenam o interior e imediações do PE Mata do Xem-Xém. 


\section{METODOLOGIA}

\section{Área de estudo}

O Parque Estadual Mata do Xem-Xém é localizado no município de Bayeux, na região metropolitana de João Pessoa, Estado da Paraíba, com área de extensão de 182 hectares. O PE Mata do Xém-Xém possui parte de seus limites em uma área de nascentes do rio do Meio, um afluente do rio Paraíba do Norte. A Lei n 7.754, de 14 de abril de 1989 estabelece as diretrizes para proteção das florestas estabelecidas nas nascentes dos rios. Essas regiões de nascentes são consideradas Áreas de Preservação Permanente (APPs), portanto é importante que estejam dentro o limite deste Parque Estadual.

\section{Amostragem}

Foram realizadas duas campanhas de coletas, que ocorreram entre os dias 19 e 20 de dezembro de 2016 e 14 e 15 de março de 2017. Nestas amostragens foram selecionados sete pontos, sendo seis em rios (dois no rio Marés - Io1 e Ioz e quatro no riacho do Meio - Io4, Io5, Io6 e Io7) e um lago (lago do Xem-Xém - Io3) (Figura 1 e 2; Tabela I).

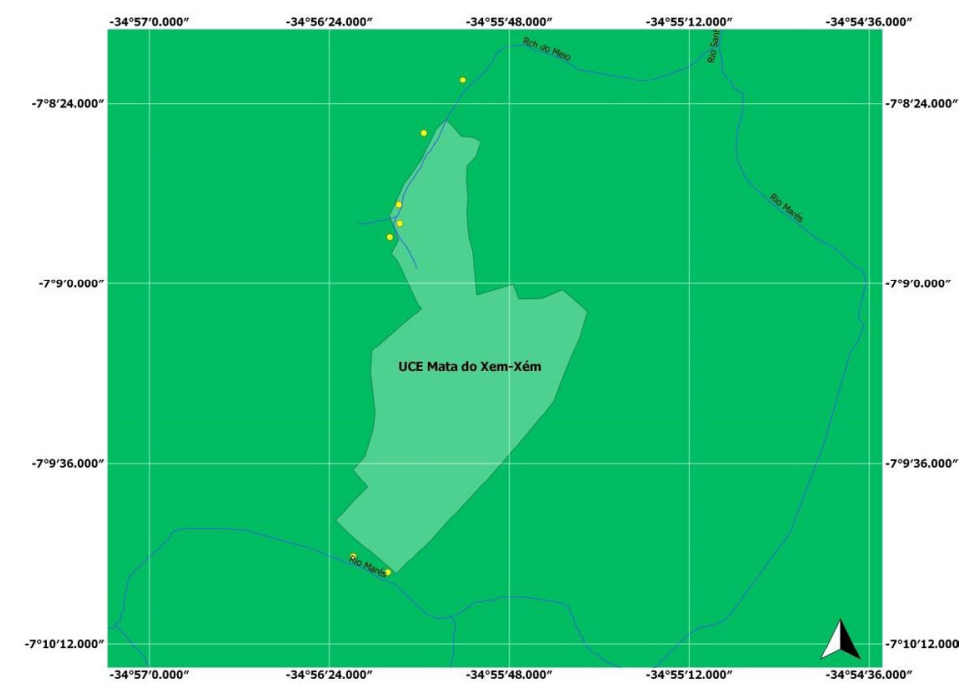

Figura 1: Mapa de distribuição dos pontos de amostragens nos corpos d'água do PE Mata de Xem-Xém, Bayeux, Paraíba.

A coleta de dados da ictiofauna do PE Mata de Xem-Xém foi realizada utilizando-se arrastos manuais (redes de arrastos de $10 \mathrm{~m}$ e $4 \mathrm{~m}$, ambas com malha de $5 \mathrm{~mm}$ ), tarrafa (malha de $20 \mathrm{~mm}$ ), puçás (malhas de $5 \mathrm{~mm}$ ) e rede de espera (malha $20 \mathrm{~mm})$. 

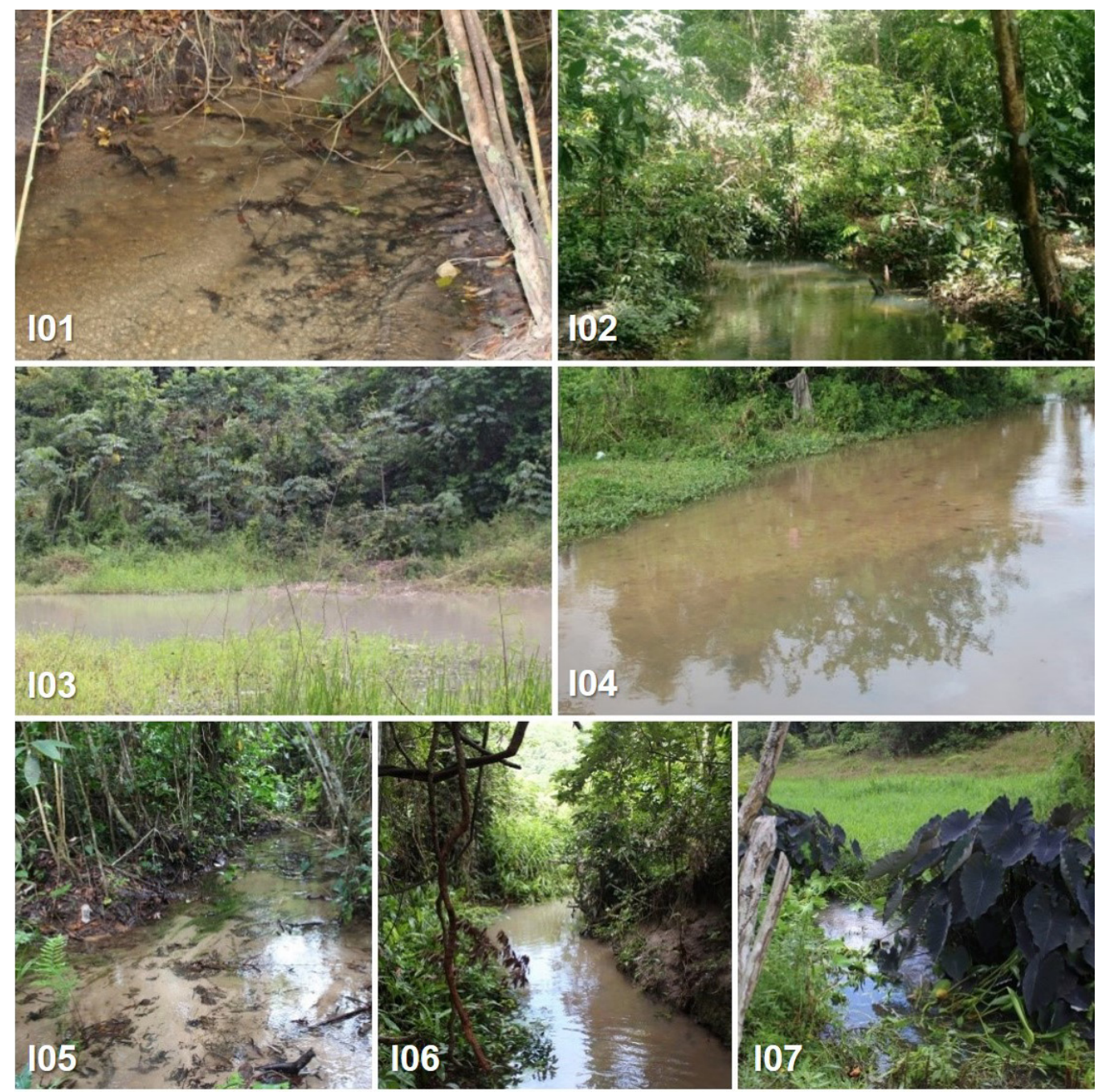

Figura2: Pontos amostrados na coleta de peixes de água doce do PE Mata do Xem-Xém, Bayeux, Paraíba. Io1: Rio Marés (1); Io2: Rio Marés (2); Io3: Lago Xem-Xém; Io4: Riacho do

Meio (1); Io5: Riacho do Meio (2); Io6: Riacho do Meio (3) E Io7: Riacho do Meio (4)

Tabela I. Lista e descrição dos pontos de amostragens da ictiofauna nos corpos d'água do PE Mata de Xem-Xém, Bayeux, Paraíba

\begin{tabular}{ccc}
\hline $\begin{array}{c}\text { Ponto } \\
\text { amostral }\end{array}$ & Caracterização/Habitat & Coordenadas \\
\hline
\end{tabular}

Rio Marés (1), PE Mata do Xem-Xém; local de coleta apresenta leito com agua transparente, substrato cascalho-arenoso com bastante

Io1 folhiço, pouca vegetação aquática submersa e flutuante; bastante vegetação marginal terrestre e pouca aquática; largura em torno de 4 07 $7^{\circ}$ ' $57.7^{\prime \prime} \mathrm{S}$ $\mathrm{m}$, profundidade máxima de $1 \mathrm{~m}$ (Figura 2).

Rio Marés (2), PE Mata do Xem-Xém; local de coleta apresenta agua transparente, substrato arenoso, algumas porções com cascalho, pouca vegetação aquática submersa e flutuante; bastante vegetação marginal terrestre e pouca aquática; largura em torno de $2 \mathrm{~m}$, profundidade $034^{\circ} 56^{\prime} 12.3$ "W máxima de $80 \mathrm{~cm}$. (Figura 3). 


\begin{tabular}{|c|c|c|}
\hline $\begin{array}{c}\text { Ponto } \\
\text { amostral }\end{array}$ & Caracterização/Habitat & Coordenadas \\
\hline Io3 & $\begin{array}{l}\text { Lago Xem-Xém, PE Mata do Xem-Xém; local de coleta apresenta agua } \\
\text { barrenta, substrato argilo-arenoso, algumas porções com cascalho, } \\
\text { moderada vegetação aquática submersa e flutuante; bastante vegetação } \\
\text { marginal terrestre e aquática; largura em torno de } 10 \text { m, profundidade } \\
\text { máxima de } 2 \text { m (Figura 4). }\end{array}$ & $\begin{array}{c}07^{\circ} 08^{\prime} 48,0^{\prime \prime} \mathrm{S} \\
034^{\circ} 56^{\prime} \mathrm{og}, 9 \mathrm{~W}\end{array}$ \\
\hline Io4 & $\begin{array}{l}\text { Riacho do Meio (1), PE Mata do Xem-Xém, local de coleta apresenta } \\
\text { agua transparente, substrato cascalho-arenoso, pouca vegetação } \\
\text { aquática submersa e flutuante; moderada vegetação marginal terrestre } \\
\text { e pouca aquática; largura em torno de } 3 \mathrm{~m} \text {, profundidade máxima de } \\
80 \mathrm{~cm} \text { (Figura 5). }\end{array}$ & $\begin{array}{c}\text { o7 }{ }^{\circ} 08^{\prime} 19,3 S^{\prime \prime} \\
034^{\circ} 55^{\prime} 57,3 \text { "W }\end{array}$ \\
\hline Io5 & $\begin{array}{l}\text { Riacho do Meio (2), PE Mata doXem-Xém, apresenta água transparente, } \\
\text { substrato cascalho-arenoso-argiloso, bastante vegetação aquática } \\
\text { submersa, pouca flutuante; bastante vegetação marginal terrestre } \\
\text { cobrindo todo o ponto de amostragem, formando uma mata de galeria } \\
\text { fechada e pouca aquática; largura em torno de } 5 \mathrm{~m} \text {, profundidade } \\
\text { máxima de } 80 \mathrm{~cm} \text { (Figura 6). }\end{array}$ & $\begin{array}{c}07^{\circ} 08^{\prime} 50,7^{\prime \prime} \mathrm{S} \\
034^{\circ} 56^{\prime} 11,9^{\prime \prime} \mathrm{W}\end{array}$ \\
\hline Io6 & $\begin{array}{l}\text { Riacho do Meio (3), PE Mata do Xem-Xém, apresenta agua transparente, } \\
\text { substrato cascalho-arenoso, bastante vegetação aquática submersa e } \\
\text { pouca flutuante; bastante vegetação marginal terrestre formando uma } \\
\text { mata de galeria fechada e pouca vegetação aquática; largura em torno } \\
\text { de } 3 \mathrm{~m} \text {, profundidade máxima de } 1 \mathrm{~m} \text { (Figura } 7 \text { ). }\end{array}$ & $\begin{array}{c}07^{\circ} 08^{\prime} 44,2^{\prime \prime} \mathrm{S} \\
\mathrm{o} 34^{\circ} 56^{\prime} 10,1^{\prime \prime} \mathrm{W}\end{array}$ \\
\hline Io7 & $\begin{array}{l}\text { Riacho do Meio (4), PE Mata doXem-Xém, apresentaagua transparente, } \\
\text { substrato cascalho-arenoso, pouca vegetação aquática submersa e } \\
\text { flutuante; moderada vegetação marginal terrestre e pouca aquática; } \\
\text { largura em torno de } 2 \mathrm{~m} \text {, profundidade máxima de } 80 \mathrm{~cm} \text { (Figura } 8 \text { ). }\end{array}$ & $\begin{array}{c}07^{\circ} 08^{\prime} 29,9 \text { " } \mathrm{S} \\
034^{\circ} 56^{\prime} 05,1^{\prime \prime} \mathrm{W}\end{array}$ \\
\hline
\end{tabular}

\section{Procedimentos de Campo e Laboratório}

Os espécimes testemunhos foram anestesiados com solução de eugenol diluído em álcool e água, em seguida fixados em formalina a 10\%. Os espécimes foram tratados de acordo com as normas de curadoria científica, que consistem na sua fixação em formol durante um período mínimo de oito dias, na transferência deles para uma solução alcoólica a $75^{\circ} \mathrm{GL}$, na triagem por lotes de espécimes e na etiquetagem individual de cada lote, de acordo com Malabarba e Reis (1987). A triagem e a identificação dos espécimes foram realizadas no Laboratório de Sistemática e Ecomorfologia de Peixes da Universidade Federal da Paraíba (LASEP/ UFPB), o material coletado foi depositado, após identificação, na Coleção Ictiológica do Departamento de Sistemática e Ecologia, da mesma instituição.

Os dados merísticos e morfométricos envolvidos no processo de identificação foram tomados segundo os métodos de Hubbs e Lagler (2006), com o auxílio de microscópio estereoscópico e paquímetro. A identificação foi procedida de acordo com Ramos et al. 2018. A composição taxonômica da ictiofauna registrada foi apresentada como uma lista sistemática que seguiu Fricke et al. (2019). A Lista Nacional Oficial de 
Espécies da Fauna Ameaçada de Extinção: Peixes e Invertebrados Aquáticos (MMA 2014) foi utilizada como fonte de identificação de possíveis espécies ameaçadas de extinção.

\section{RESULTADOS}

Um total de 905 espécimes foram coletados, distribuídos em quatro ordens, seis famílias, 13 gêneros e 15 espécies (Tabela II).

Tabela II. Lista de espécies coletadas no PE Mata do Xem-Xém, com seus respectivos nomes populares, habitats e vouchers. Onde (i) refere-se a espécies Introduzidas na Bacia do Rio Paraíba

Taxon

Nome Popular

Habitat Voucher

ORDEM CHARACIFORMES

Família Characidae

\begin{tabular}{llll}
\hline Astyanax bimaculatus (Linnaeus, 1758) & Piaba & Dulcícola & UFPB-11171 \\
\hline Cheirodon jaguaribensis Fowler, 1941 & Piaba & Dulcícola & UFPB-11194 \\
\hline Hemigrammus marginatus Ellis, 1911 & Piaba & Dulcícola & UFPB-11192 \\
\hline Hemigrammus rodwayi Durbin, 1909 & Piaba & Dulcícola & UFPB-11195 \\
\hline Hyphessobrycon parvellus Ellis, 1911 & Piaba & Dulcícola & UFPB-11179 \\
\hline Serrapinnus piaba (Lütken, 1875) & Piaba & Dulcícola & UFPB-11173 \\
\hline Família Erythrinidae & & & \\
\hline Hoplias malabaricus (Bloch, 1794) & Traíra & Dulcícola & UFPB-11184 \\
\hline
\end{tabular}

ORDEM SILURIFORMES

Família Heptapteridae

Rhamdia quelen (Quoy \& Gaimard 1824)

Jundiá

Dulcícola UFPB-11172

ORDEM CYPRINODONTIFORMES

Família Poeciliidae

\begin{tabular}{llll}
\hline Poecilia vivipara Bloch \& Schneider, 1801 & Guarú, Guru & Dulcícola & UFPB-11183 \\
\hline Poecilia reticulata Peters, $1859^{(\mathrm{I})}$ & Guarú, Gupi & Dulcícola & UFPB-11184
\end{tabular}

ORDEM CICHLIFORMES

Família Cichlidae

\begin{tabular}{llll}
\hline Geophagus brasiliensis Kner, 1865 & Cará & Dulcícola & UFPB-11170 \\
\hline Cichlasoma orientale Kullander, 1983 & Cará-preto & Dulcícola & UFPB-11180 \\
\hline Crenicichla brasiliensis (Bloch, 1792) & Jacundá & Dulcícola & UFPB-11185 \\
\hline Oreochromis niloticus (Linnaeus, 1758) & Tilápia & Dulcícola & UFPB-11186 \\
\hline Família Eleotridae & & & \\
\hline Eleotris pisonis (Gmelin,1789) & Moré Preta & $\begin{array}{l}\text { Estuarino- } \\
\text { Marinho }\end{array}$ & UFPB-11191 \\
\hline
\end{tabular}


Entre às 15 registradas, sete pertencem à ordem Characiformes, o que representa $46.7 \%$ do total de espécies. As espécies pertencentes a essa ordem estão distribuídas em seis gêneros e duas famílias. Não foram encontradas espécies de Characiformes introduzidas na amostragem. A segunda ordem mais representativa foi Cichliformes, com cinco espécies (33.3\%), cinco gêneros e duas famílias. Das espécies de Cichliformes, uma é introduzida, a tilápia, Oreochromis niloticus. A ordem Cyprinodontiformes foi representada por duas espécies (13.3\%) do mesmo gênero, entre as duas, uma é considerada introduzida, o guaru ou barrigudinho, Poecilia reticulata. Siluriformes, foi representada por apenas uma espécie, Rhamdia quelen (6.7\%) (Figura 3).

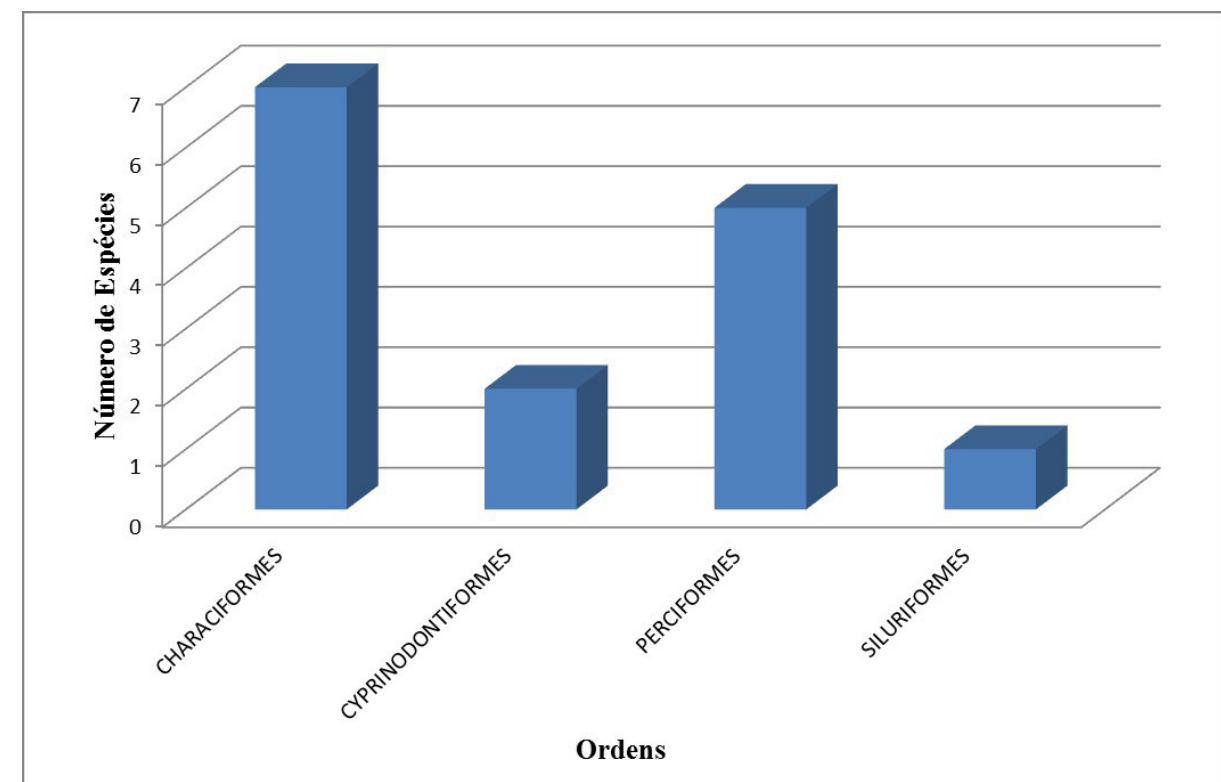

Figura 3. Número de espécies por ordem coletadas do PE Mata do Xem-Xém

As famílias com maior número de espécies foram Characidae (Characiformes) com seis espécies (40\% do total), seguida de Cichlidae (Cichliformes) com quatro (26.7\%). Das quatro espécies da família Cichlidae, três são consideradas nativas para região. Poeciliidae foi representada por duas espécies (13.4\%), uma nativa e outra exótica. As outras três famílias, Heptapteridae, Eleotridae e Erythrinidae, foram representadas por apenas uma espécie (6.6\% cada). A espécie da família Eleotridae (Eleotris pisonis) é uma espécie estuarino-marinha.

Das 15 espécies registradas no PE Mata de Xem-Xém duas são introduzidas e 13 nativas, destas 13, 12 são exclusivamente dulcícolas e uma estuarino-marinha (Tabela II). Três espécies dulcícolas são endêmicas da região Nordeste: Cheirodon jaguaribensis, Cichlasoma orientale e Crenicichla brasiliensis. Não foram registradas espécies ameaçadas, quando se compara a lista de espécies detectada no PE Mata do Xem-Xém com aquelas da Portaria MMA n²45, de 17 de dezembro de 2014, do Ministério do Meio Ambiente (MMA 2014). 
Em relação a abundância das espécies coletadas, Hemigrammus marginatus foi a mais abundante com 430 espécimes coletados (48\% dos 905 coletados), seguida de Hemigrammus rodwayi com 194 espécimes (22\%). As outras espécies apresentaram menos de 110 exemplares.

A espécie mais amplamente distribuída nos corpos d'água do PE Mata do Xem-Xém foi Crenicichla brasiliensis, sendo coletada em seis dos sete pontos de amostragens. Três espécies foram registradas em cinco pontos (Hemigrammus marginatus, Hoplias malabaricus e Poecilia reticulata), duas em quatro pontos (Astyanax bimaculatus e Poecilia vivipara). As outras espécies foram registradas em menos da metade dos pontos de amostragens.

\section{DISCUSSÃO}

Os resultados observados nos corpos d'águas do PE se encontram dentro do que já foi registrado para áreas de Mata Atlântica na ecorregião Nordeste/Caatinga. Gomes-Filho (1999) realizou o inventário das espécies de peixes, apenas da ordem Characiformes, das bacias costeiras do Estado da Paraíba: Abiaí, Camaratuba, Gramame, Jaguaribe, Paraíba do Norte e Mamanguape. Nesse estudo foram registradas 18 espécies dentro de 13 gêneros e sete famílias. Rosa e Groth (2004) realizaram um levantamento ictiofaunístico dos brejos de altitude dos estados da Paraíba e Pernambuco. Rodrigues-Filho et al. (2016) inventariou os enclaves de floresta úmida nos planaltos da Ibiapaba e do Araripe, nos estados do Ceará e Pernambuco, e elencou 59 espécies.

No trabalho de Ramos et al. (2018) para a mesma bacia foram registradas 47 espécies. Todas as espécies registradas no presente estudo para PE Mata do XemXém também foram listadas por Ramos et al. (2018). De fato, os autores parecem apresentar a lista com o número real de espécies a ocupar essa bacia hidrográfica, tendo em vista a ampla amostragem realizada.

Como citado anteriormente o PE Mata do Xem-Xém se encontra sob domínio da Mata Atlântica. O número de inventários da ictiofauna de água doce dos rios da Mata Atlântica no Nordeste Médio Oriental ainda são escassos. Torelli et al. (1997), Gomes-Filho \& Rosa (2001), e Paiva et al. (2014) realizaram levantamentos em bacias que estão com suas hidrografias completamente dentro da Mata Atlântica. No estado da Paraíba na bacia do rio Gramame, Torelli et al. (1997) registraram 22 espécies distribuídas em 22 gêneros e 15 famílias e Gomes-Filho \& Rosa (2001) registraram 32 espécies das quais 23 são de água doce. No estado do Rio Grande do Norte na microbacia do rio Pratagi, Paiva et al. (2014) elencaram 22 espécies das quais 13 são de água doce. Porém, é importante destacar que os trabalhos citados, que apresentaram maiores riquezas, foram realizados em áreas de maior extensão (bacias hidrográficas) que aquela utilizada no presente estudo, haja visto que, o foco deste foi inventariar 
as espécies que estavam sendo protegidas por uma Área de Conservação, que drena apenas uma parte da bacia do Rio Paraíba do Norte.

Porém, recentemente foi publicado um estudo de Gouveia et al. 2017, em uma reserva ambiental no município de Mamanguape (Reserva do Rio Guaribas), também no estado da Paraíba, os autores elencaram 18 espécies, número aproximado ao encontrado no presente trabalho, o qual corrobora também, com a captura das duas espécies introduzidas coletadas neste estudo (O. niloticus e $P$. reticulata), ambas dentro das áreas de proteção no estado da Paraíba.

As duas espécies do gênero Hemigrammus, registradas como as mais abundantes, são de pequeno porte, e pertencem a um grupo de peixes conhecido na região com o nome popular de "piabas" e não despertam interesse maior por parte da população. Estes peixes, muitas vezes são apenas usados por pescadores como iscas para captura de espécies maiores. Um aspecto que explica a abundância das piabas é fato desse grupo formar cardumes, o que facilita a coleta de um maior número de espécimes (Ramos 2012). Unidas estas duas espécies corresponderam a 70\% dos indivíduos coletados. Hemigrammus rodwayi, é uma espécie de localidade-tipo registrada em rios da Guyana (Reis et al. 2003), sendo atualmente também registrada em alguns rios do nordeste (Rodrigues-Filho et al. 2016, Costa et al, 2017, Ramos et al. 2018, Oliveira-Silva et al. 2018). A ocorrência dessa e outras espécies é considerada natural (não foi introduzida) por alguns pesquisadores, que explicam este fato pelas relações pretéritas da Mata Atlântica com a Amazônia (Menezes et al. 2007; Ramos et al. 2018).

É importante chamar atenção para o fato de que foram registradas duas espécies consideradas exóticas para região, a tilápia, Oreochromis niloticus e o guaru, Poecilia reticulata. Oreochromis niloticus conhecido como "tilápia do Nilo" é uma espécie natural de algumas bacias africanas e que se encontra bem estabelecida em todo o Nordeste e outras regiões brasileiras, atualmente (Leão et al. 2011, Levis et al. 2013). A tilápia apresenta uma ampla plasticidade trófica se adaptando rapidamente a maioria dos ambientes, alimentando-se principalmente na base das cadeias alimentares, é altamente tolerante às variações ambientais e apresenta rápida explosão populacional (Agostinho 1993, Gurgel e Fernando 1994, Starling et al. 2002, Attayde et al. 2007).

Peixes onívoros filtradores como a $O$. niloticus, tendem a aumentar a biomassa fitoplanctônica total, através da predação sobre o zooplâncton e da reciclagem de nutrientes que afeta as outras espécies de peixes devido a alterações na qualidade do habitat (Starling et al., 2002). Além disso, as tilápias competem com as espécies de peixes nativas, por recursos alimentares (Drenner et al. 1996, Attayde et al. 2007). Outro fator para o sucesso da espécie é a baixa taxa de mortalidade da prole, em função do cuidado parental, as tilápias desalojam outros peixes em função da agressividade com que defendem seus ninhos (Attayde et al. 2007). Portanto, a introdução de espécies exóticas, como Oreochromis niloticus, causam impactos significativos por sufocar as espécies nativas, aumentando o estresse ambiental e a competição, como consequência tende a causar diminuição na diversidade das espécies nativas (Agostinho 1993 e 1996, Buckup 1998). No entanto, no PE Mata do 
Xem-Xém foram registrados apenas três exemplares de O. niloticus. Esses poucos exemplares foram encontrados em um único ponto, no lago Xem-Xém, não sendo registrado no rio do Meio que segue a partir do açude. Portanto, é provável que essa espécie ainda não tenha se alastrado no rio.

Devido a todos os impactos supracitados que a tilápia, $O$. niloticus, pode causar, somado a fato do PE Mata do Xem-Xém conserva 13 (36.2\%) das 36 espécies nativas da bacia do rio Paraíba do Norte e pelo lago Xem-Xém está localizado em uma área de nascentes de um afluente do rio Paraíba do Norte, destacamos a importância de possíveis medidas para retirada desta espécie da UC. Isso é de fato importante se considerarmos o que consta no Art. 31 da Lei Federal $N^{\circ}$ 9.985/20oo que proíbe a introdução nas unidades de conservação de espécies não autóctones.

A outra espécie exótica é o guaru ou barrigudinho, Poecilia reticulata, tem localidade-tipo registrada para Caracas, Venezuela, e ampla distribuição na América do Sul e Ilhas do Caribe: nas bacias costeiras da Venezuela, a leste de Maracaibo, Barbados, Trinidade, norte do Brasil e nas Guianas (Lucinda 2003). É uma espécie de pequeno porte, tendo como principal motivo de sua introdução o uso para fins de controle de insetos, como Aedes aegypti, no combate à dengue (Lucinda 2003, Levis et al. 2013). No Nordeste $P$. reticulata foi introduzida pelo Departamento Nacional de Obras Contra as Secas (DNOCS) na década de 1920 para o combate ao mosquito da febre amarela (Shibatta et al. 2002).

Outro motivo da introdução desta espécie é devido ao seu uso no aquariofilismo. Os machos de $P$. reticulata exibem o colorido bastante chamativo, o que desperta muito a atenção dos aquariofilistas, que quando desistem do cultivo, muitas vezes podem jogá-las em córregos e riachos. As espécies do gênero Poecilia são geralmente bastante prolíferas, são vivíparas, apresentam cuidado parental, esses aspectos contribuem bastante para o crescimento populacional (Levis et al. 2013). P. reticulata na UCE Mata de Xem-Xém foi a terceira espécie mais abundante com 86 espécimes coletados (10.7\% da amostragem), além disso, também foi uma das duas únicas registradas em todos os pontos de amostragens. Portanto, esta espécie já se estabeleceu no ambiente, o que torna impraticável a sua retirada da UCE Mata de Xem-Xém. Os impactos que essa espécie pode causar no ambiente e as outras espécies nativas ainda estão sendo estudados (Magalhães 2008).

Como citado acima, o PE Mata do Xem-Xém possui a região Noroeste de seus limites em uma área de nascentes do rio do Meio, que é um afluente do rio Paraíba do Norte. A Lei ${ }^{\circ} 7.754$, de 14 de abril de 1989 estabelece as diretrizes para proteção das florestas estabelecidas nas nascentes dos rios. Essas regiões de nascentes são consideradas Áreas de Preservação Permanente (APPs), portanto, seria muito importante para conservação desse manancial, que o limite deste Parque Estadual abrangesse toda a região de nascentes. A partir desse e outros estudos (Gouveia et al. 2017), que vem sendo desenvolvidos podemos constatar a vulnerabilidade desses ecossistemas ao estabelecimento de espécies invasoras e possíveis impactos futuros que estas possam vir a causar para a fauna endêmica da região. Dessa forma, faz-se 
de grande importância a implementação de Planos de conservação e manejo para essas áreas de preservação que encontram-se dentro do bioma Mata Atlântica.

\section{AGRADECIMENTOS}

Agradecemos ao Professor Dr. Bráulio Araújo Almeida Santos pelas discussões envolvendo Conservação, a Raizze Avelar pelo auxílio na coleta e triagem dos espécimes. O presente trabalho foi realizado com apoio da Coordenação de Aperfeiçoamento de Pessoal de Nível Superior - Brasil (CAPES) - Código de Financiamento oo1. A Empresa Office o4 Consultoria Ambiental pelo apoio logístico que tornou este estudo possível.

\section{REFERÊNCIAS}

Abell R, Thieme M L, Revenga C, Bryer M, Kottelat M, Bogutskaya N, Coad B, Mandrak N, Contreras-Balderas S, Bussing W, Stiassny M L J, Skelton P, Allen G R, Unmack P, Naseka A, Ng R, Sindorf N, Robertson J, Armijo E, Higgins J V, Heibel T J, Wikramanayake E, Olson D, López H L, Reis R E, Lundberg J G, Sabaj-Pérez M H, Petry P. 2008. Freshwater ecoregions of the world: a new map of biogeographic units for freshwater biodiversity conservation. Bioscience 58(5):403-414.

Agostinho A A. 1993. Considerações sobre a ictiofauna das principais bacias hidrográficas brasileiras. Anais do Xo Encontro Brasileiro de Ictiologia, 301.

Agostinho A A. 1996. Ameaça Ecológica: Peixes de outras águas. Revista Ciência Hoje. Vol.21/n ${ }^{\circ} 124$. p. 36-44.

Albagli S. 2001. Amazônia: Fronteira Geopolítica de Biodiversidade. Parcerias estratégicas, n. 12, p. 5-9.

Attayde J L, Okun N, Brasil J, Menezes R, Mesquita P. 2007. Os impactos da introdução da tilápia do Nilo, Oreochromis niloticus, sobre a estrutura trófica dos ecossistemas aquáticos do Bioma Caatinga. Oecologia Brasiliensis, 11(3), 450-461.

Buckup P A. 1998. A piscicultura de espécies exóticas e problemas ecológicos. $A$ Natureza em Revista. pp. 20-23.

Costa S Y L., Viana L G, Barbosa J E L, Ramos T P A. 2017. Composition of the ichthyofauna in Brazilian semiarid reservoirs. Biota Neotropica. 17(3): e20170334 
Drenner R W, Smith J D, Threlkeld S T. 1996. Lake trophic state and the limnological effects of omnivorous fish. Hydrobiologia, 319(3), 213-223.

Fricke R, Eschmeyer W N, Van der Laan R. 2019. ESCHMEYER'S CATALOG OF FISHES: GENERA,SPECIES,.(http://researcharchive.calacademy.org/research/ichthyology/ catalog/fishcatmain.asp). Electronic version accessed 18 Mai 2019.

Gomes-Filho G, Rosa S R. 2001. Inventário da Ictiofauna da Bacia do Rio Gramame, Paraíba, Brasil. In: A Bacia do Rio Gramame: Biodiversidade, Uso e Conservação. Watanabe, T (ed.). João Pessoa: Prodema, p 167-173.

Gomes-Filho G. 1999. Characiformes (Actinopterygii: Ostariophysi) das Bacias Costeiras do Estado da Paraíba. João Pessoa: Departamento de Sistemática e Ecologia/ UFPB. 1999. $90 \mathrm{p}$.

Gouveia R S D, Lira G L A, Ramos T P A, Medeiros E S F. 2017. Ichthyofauna of the Reserva Biológica Guaribas and surrounding areas, state of Paraíba, Brazil. Check List. 13(5): 581-590. https://doi. org/10.1556o/13.5.581.

Gurgel J J S, Fernando C H. 1994. Fisheries in Semi-Arid Northeast Brazil with Special Reference to the Role of Tilapias. Internationale Revue der gesamten Hydrobiologie und Hydrographie, 79(1), 77-94.

Hubbs C L, Lagler K F. 2006. Fishes of the Great Lakes region. Ann Arbor: University of Michigan Press. p.1-213.

Leão T C, Almeida W D, Dechoum M D E S, Ziller S R. 2011. Espécies exóticas invasoras no Nordeste do Brasil: contextualização, manejo e políticas públicas. Centro de Pesquisas Ambientais do Nordeste e Instituto Hórus de Desenvolvimento e Conservação Ambiental. Recife, PE, 33.

Levis C, Ramos T P A, Lima S M Q. 2013. A disputa desigual entre peixes nativos e exóticos do semiárido. Natal: EDUFRN. 67p.

Lira G L A. 2015. Ictiofauna da bacia do rio Abiaí, Nordeste, Brasil. João Pessoa: Centro de Ciências Biológicas e Sociais Aplicadas/UEPB. p.58 .

Lucinda P. H. 2003. Family Poeciliidae. Check list of the freshwater fishes of South and Central America, 555, 581.

Magalhães A L B. 20o8. O gupi Poecilia reticulata Peters (Osteichthyes, Poeciliidae) introduzido na Serra do Espinhaço, Minas Gerais. Boletim da Sociedade Brasileira de Ictiologia, 92, 5-7. 
Malabarba L R, Reis R E. 1987. Manual de técnicas para a preparação de coleções zoológicas. Sociedade Brasileira de Zoologia, Campinas, v. 36, p. 1-14.

Menezes N A, Weitzman S H, Oyakawa O T, Lima F C T D, Correa e Castro R M, Weitzman M J. 2007. Peixes de água doce da Mata Atlântica: lista preliminar das espécies e comentários sobre conservação de peixes de água doce neotropicais. São Paulo: Museu de Zoologia da Universidade de São Paulo. 407 pp.

Ministério Do Meio Ambiente. 2014. Lista Nacional do Risco de Extinção da Fauna Brasileira. Instituto Chico Mendes da Biodiversidade (Portaria MMA n 445, de 17 de dezembro de 2014).

MMA. 2019. Unidade de Conservação. Sistema Nacional de Conservação da Natureza. Disponível em: http://www.mma.gov.br/areas-protegidas/unidades-de-conservacao. Acesso em 18/05/2019.

Odum E P. 1988. Ecologia. Rio de Janeiro: Guanabara Koogan.

Oliveira-Silva L, Ramos T P A, Carvalho-Rocha Y G P, Viana K M P, Avellar R C, Ramos R T C. 2018. Ichthyofauna of the Mamanguape river basin, Northeastern, Brazil. Biota Neotropica. 18(3): e20170452.

Paiva R E C, Lima S M Q, Ramos T P A, Mendes L. 2014. Fish fauna of Pratagi River coastal microbasin, extreme north Atlantic Forest, Rio Grande do Norte State, northeastern Brazil. Check List (São Paulo. Online), v. 10, p. 968-975.

Pinto L P, Hirota M, Guimarães E, Fonseca M, Martinez D I, Takahashi C K. 2018. Unidades de Conservação Municipais da Mata Atlântica. Relatório Técnico. São Paulo: Fundação SOS Mata Atlântica.

Ramos T P A, Lima J A S, Costa S Y L, Silva M J, Avellar R C, Oliveira-Silva L. 2018. Continental ichthyofauna from the Paraíba do Norte River basin pre-transposition of the São Francisco River, Northeastern Brazil. Biota Neotropica. 18(4): e20170471.

Ramos T P A. 2012. Ictiofauna de Água Doce da Bacia do rio Parnaíba. João Pessoa: Departamento de Sistemática e Ecologia/UFPB. 2012. 434 p. (Tese de Doutorado).

Reis R E, Kullander S O, Ferraris J, Carl J. 2003. Check List of the freshwater fishes of South and Central America. Porto Alegre: EDIPUCRS. 729p.

Rodrigues-Filho C A S, Gurgel-Lourenco R C, Bezerra L A V, Sousa W A, Garcez D S, Lima S M Q, Ramos T P A, Botero J I S. 2016. Ichthyofauna of the humid forest 
enclaves in the tablelands of Ibiapaba and Araripe, Northeastern Brazil. Biota Neotropica (Online. Edição em Inglês), v. 14, p. e20160273.

Rosa R S, Groth F. 2004. Ictiofauna dos Ecossistemas de Brejos de Altitude de Pernambuco e Paraíba. In: Kátia C Porto; J.J.P. Cabral; p.201-210

Sá-Neto AA. 2004. Levantamento da Ictiofauna de Água Doce da bacia do rio Jaguaribe, João Pessoa, PB. João Pessoa: Departamento de Sistemática e Ecologia/UFPB. 2004. 3op. (Monografia).

Secretaria Extraordinária Do Meio Ambiente Dos Recursos Hídricos E Minerais Da Paraíba - SEMARH. 2016. Plano Estadual de Recursos Hídricos da Paraíba-PERH-PB. Resumo Executivo e Atlas. João Pessoa.

Shibatta O A, Orsi M L, Bennemann S T, Silva-Souza A T. 2002. Diversidade e distribuição de peixes na bacia do rio Tibagi, pp. 403-423.

Starling F, Lazzaro X, Cavalcanti C, Moreira R. 2002. Contribution of omnivorous tilapia to eutrophication of a shallow tropical reservoir: evidence from a fish kill. Freshwater Biology, 47: 2443-2452.

Tabarelli M, Pinto LP, Silva J M C, Hirota M M, Bedê LC. 2005. Desafios e oportunidades para a conservação da biodiversidade na Mata Atlântica brasileira. Megadiversidade, $1(1), 132-138$.

Torelli J, Rosa I L, Watanabe T. 1997. Ictiofauna do Rio Gramame, Paraíba, Brasil. Iheringia, sér. Zool., 82:67-73. 